\title{
Charge ordering and phase separation in the infinite dimensional extended Hubbard model
}

\author{
Ning-Hua Tong, ${ }^{1}$ Shun-Qing Shen, ${ }^{2}$ and Ralf Bulla ${ }^{1}$ \\ ${ }^{1}$ Theoretical Physics III, Center for Electronic Correlations and Magnetism, Institute of Physics, University of Augsburg, \\ D-86135 Augsburg, Germany \\ ${ }^{2}$ Department of Physics, The University of Hong Kong, Pokfulam, Hong Kong, China
}

(Received 1 March 2004; revised manuscript received 27 May 2004; published 31 August 2004)

\begin{abstract}
We study the extended Hubbard model with both on-site $(U)$ and nearest neighbor $(V)$ Coulomb repulsion using the exact diagonalization method within the dynamical mean field theory. For a fixed $U(U=2.0)$, the $T-n$ phase diagrams are obtained for $V=1.4$ and $V=1.2$, at which the ground state of $n=1 / 2$ system is chargeordered and charge-disordered, respectively. In both cases, robust charge order is found at finite temperature and in an extended filling regime around $n=1 / 2$. The order parameter changes nonmonotonously with temperature. For $V=1.4$, phase separation between charge-ordered and charge-disordered phases is observed in the low temperature and $n<0.5$ regime. It is described by an "S"-shaped structure of the $n-\mu$ curve. For $V$ $=1.2$, the ground state is charge-disordered, and a reentrant charge-ordering transition is observed for $0.42<n<0.68$. The relevance of our results to experiments for doped manganites is discussed.
\end{abstract}

DOI: $10.1103 /$ PhysRevB.70.085118

PACS number(s): 71.27.+a, 71.45.Lr, 64.75.+g

\section{INTRODUCTION}

Charge-ordering is a fascinating research topic in condensed matter physics. In recent years, charge order and the related spin and orbital order in doped manganites have attracted much interest. In the system $\mathrm{La}_{1-x} \mathrm{Ca}_{x} \mathrm{MnO}_{3}(x>0.5)$, charge order in the ground state is enhanced as $x$ increases. The corresponding transition temperature $T_{\mathrm{co}}$ is higher than the Néel temperature, ${ }^{1}$ and there are strong charge-order fluctuations at temperatures above $T_{\text {со }}$ (Ref. 2). In particular, a reentrant charge-ordering transition has been observed in systems such as $\operatorname{Pr}_{0.65}\left(\mathrm{Ca}_{0.7} \mathrm{Sr}_{0.3}\right)_{0.35} \mathrm{MnO}_{3}$ (Ref. 3) and $\mathrm{LaSr}_{2} \mathrm{Mn}_{2} \mathrm{O}_{7}$ (Refs. 4 and 5). The origin of these chargeordering transitions lies in the complex interplay between orbital and lattice degrees of freedom in the manganites, and is presently under intensive study. In other systems such as the heavy fermion system $\mathrm{Yb}_{4} \mathrm{As}_{3}$ (Ref. 6), the quasi-onedimensional material $\mathrm{NaV}_{2} \mathrm{O}_{5}$ (Refs. 7 and 8), and the superconducting layered organic molecular crystal $\kappa-(B E D T$ $-T T F)_{2} X$ (Ref. 9), charge-ordering is closely related to the specific properties of the system.

The charge ordering in the above stated systems has different physical origins, and cannot be explained within a single theory. From a theoretical point of view, an obvious cause of charge-ordering is the short-range Coulomb repulsion between electrons. The simplest model that includes this interaction is the extended Hubbard model which contains the kinetic term and the on-site and nearest-neighbor Coulomb repulsion. Despite the simplicity of this model, recent studies found that it can explain some characteristics of the experimental observations in doped manganites. ${ }^{10-12}$ These studies revealed that many interesting effects arise simply from pure Coulomb repulsion and charge fluctuations. In particular, using dynamical mean-field theory (DMFT), ${ }^{13}$ Pietig et al. found that the quarter-filled extended Hubbard model exhibits a reentrant charge-ordering transition near a critical value $V_{c}$ of the nearest-neighbor repulsion ${ }^{11}$ (Fig. 1). It coincides with what was observed in the doped manganites
$\mathrm{Pr}_{0.65}\left(\mathrm{Ca}_{0.7} \mathrm{Sr}_{0.3}\right)_{0.35} \mathrm{MnO}_{3}$ (Ref. 3) and $\mathrm{LaSr}_{2} \mathrm{Mn}_{2} \mathrm{O}_{7}$ (Refs. 4 and 5). This indicates that some properties of charge order may be independent of the concrete microscopic mechanism, and hence can be studied using simplified models such as the quarter-filled extended Hubbard model. Along this line, other authors also studied this problem. ${ }^{14-17}$ In lower dimensions, the model has also been extensively studied in various contexts using different methods. ${ }^{18}$

Experimentally, in the doped manganites, charge-ordering was observed not only at $x=0.5$, but also in a broad doping regime. Also the reentrant behavior was observed in systems away from quarter-filling. In fact, $\mathrm{Pr}_{0.65}\left(\mathrm{Ca}_{0.7} \mathrm{Sr}_{0.3}\right)_{0.35} \mathrm{MnO}_{3}$ has an electron filling of $n=0.65>0.5$. Another feature of the experimental observation is the inhomogeneous coexistence of charge-ordered and charge-disordered phases. In the system $\mathrm{R}_{1-x} \mathrm{Ca}_{x} \mathrm{MnO}_{3}(\mathrm{R}=\mathrm{La}, \mathrm{Nd}, \mathrm{Bi}$, etc.), many experiments showed that phase separation (PS) between a ferromagnetic charge-disordered phase and an antiferromagnetic chargeordered phase exists at dopings ranging from $x=0.33$ (Ref. 19) to $x=0.82$ (Ref. 20). Although detailed explanations should take into account the spin and orbital degrees of freedom, simplified models also give remarkably similar results.

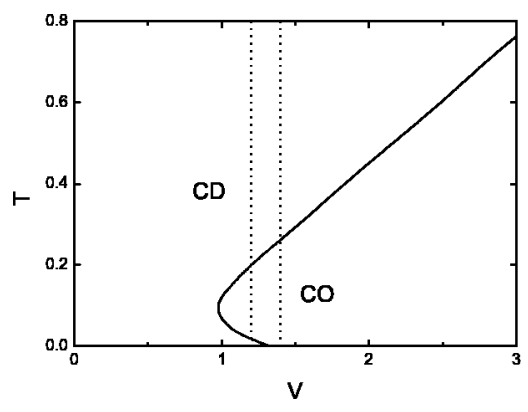

FIG. 1. Schematic $T$ - $V$ phase diagram for the extended Hubbard model at $U=2.0$ and $n=1 / 2$ (taken from Ref. 11, with $V$ rescaled.). $\mathrm{CO}$ and $\mathrm{CD}$ denote charge-ordered and charge-disordered, respectively. The vertical dashed lines mark out the positions of $T-n$ planes studied in this paper, i.e., $V=1.2$ and $V=1.4$, respectively. 
In the spinless fermion model, when electrons (holes) are added to a half-filled system $(n=1 / 2)$, an electron (hole) rich charge-disordered phase is separated from the chargeordered background. ${ }^{21}$

In this paper, motivated by the experimental observations as well as by the work of Pietig et al. ${ }^{11}$ we study the properties of a single-band extended Hubbard model away from but near quarter-filling. We confine our study to the twosublattice case and do not consider a possible incommensurate ordering. We are interested in the following two issues of charge-ordering. First, the stability of the reentrant chargeorder transition in the whole doping regime. Second, the possibility of PS between charge-disordered and charge-ordered phases. These issues are relevant to the charge-ordering and PS phenomena observed in doped manganites. Theoretical studies in the past focused only on fillings at or near $n=1.0$ and $n=0.5$. Despite the intensive studies, these two issues remain unclear.

Using DMFT together with the exact diagonalization technique, we study the paramagnetic phase diagram in two $T$ - $n$ planes for a fixed $U$-value $U=2$. As shown in Fig. 1, these two planes cross the $T-V$ plane at $V=1.2$ and $V=1.4$, which are larger and smaller than $V_{c}$, respectively $\left[V_{c}\right.$ is the critical value of the nearest-neighbor repulsion for a quarterfilled system, which separates charge-ordered and chargedisordered ground states. $V_{c} \approx 1.32 \mathrm{~W}$ (Ref. 11), see Fig. 1]. We found that a reentrant charge-ordering transition exists in an extended regime of the electron density $n$ near quarterfilling.

For $V>V_{c}$, the order-parameter of charge-ordering changes nonmonotonously as temperature decreases. The ground state is still charge-ordered for $n>0.5$ regime and charge-disordered for $n<0.5$. In the former regime, we also find the PS between charge-ordered and charge-disordered phases.

For $V$ a little smaller than $V_{c}$, the ground state is chargedisordered for any $n$. The charge-ordering exists only at finite temperatures and is most robust in the $n>0.5$ regime. At the lower critical temperature of the reentrant transition, the order-parameter disappears rather abruptly. Phase diagrams in these two planes are plotted.

In Sec. II, we describe the model and the method used in this work. In Sec. III, our results for $V=1.4 \mathrm{~W}>V_{c}$ are presented. Phase separation between charge-ordered and chargedisordered phases are discussed. Their thermodynamical structure is compared with other kinds of PSs. Our results for $V=1.2 \mathrm{~W}<V_{c}$ are presented in Sec. IV. A summary is given in Sec. V.

\section{MODEL AND METHOD}

The Hamiltonian of the single-band extended Hubbard model has the form

$$
H=-t \sum_{\langle i, j\rangle \sigma} c_{i \sigma}^{\dagger} c_{j \sigma}+U \sum_{i} n_{i \uparrow} n_{i \downarrow}+V \sum_{(i, j)} n_{i} n_{j}-\mu \sum_{i} n_{i} .
$$

In Eq. (1), $\Sigma_{\langle i, j\rangle}$ indicates the sum over nearest-neighbor sites $i$ and $j$ independently. $\Sigma_{(i, j)}$ indicates the sum over nearest pairs. Hence, there is the relation $\Sigma_{(i, j)}=1 / 2 \Sigma_{\langle i, j\rangle} . U$ and $V$ are on-site and nearest-neighbor Coulomb repulsion, respectively, and $\mu$ is the chemical potential. We use a Bethe lattice which produces a semicircular density of states for free electrons in the limit of large coordination number. In this limit, the intersite coupling terms in Eq. (1) are replaced by the corresponding Hartree term. After a proper rescaling of $V$ : $V \rightarrow V / Z$ (here $Z$ is the number of nearest neighbors) and neglecting the constant term, we obtain the following meanfield Hamiltonian:

$$
H_{\mathrm{mf}}=-t \sum_{\langle i, j\rangle \sigma} c_{i \sigma}^{\dagger} c_{j \sigma}+U \sum_{i} n_{i \uparrow} n_{i \downarrow}-\sum_{i}\left(\mu-V\left\langle n_{i+\delta}\right\rangle\right) n_{i},
$$

where $i+\delta$ is the nearest site of $i$. Note that our scaling is different from that of Ref. 11, where the scaling $V \rightarrow 2 V / Z$ is used. Therefore in this paper the value of $V$ is twice as large as the corresponding one in Ref. 11.

To describe the charge-ordered phase, we divide the Bethe lattice into two sublattices. Correspondingly, within DMFT, the model Eq. (2) is mapped onto two uncoupled effective Anderson impurity models:

$$
\begin{aligned}
H_{\Lambda, \text { imp }}= & \sum_{k=1, \sigma}^{N_{S}-1}\left[\epsilon_{\Lambda k} a_{\Lambda k \sigma}^{\dagger} a_{\Lambda k \sigma}+V_{\Lambda k}\left(a_{\Lambda k \sigma}^{\dagger} c_{\Lambda \sigma}+\text { h.c. }\right)\right] \\
& +U n_{\uparrow}^{c} n_{\downarrow}^{c}-\left(\mu-V\left\langle n_{\bar{\Lambda}}^{c}\right\rangle\right) n_{\Lambda}^{c} .
\end{aligned}
$$

Where $\Lambda=A, B$ refers to the two sublattices $(\bar{A}=B, \bar{B}=A)$, and $\left\{\varepsilon_{\Lambda k}, V_{\Lambda k}\right\}$ are effective parameters describing the bath. They are related to the Weiss function $\mathcal{G}_{\Lambda 0}^{-1}\left(i \omega_{n}\right)$ through

$$
\mathcal{G}_{\Lambda 0}^{-1}\left(i \omega_{n}\right)_{\text {map }}=i \omega_{n}+\left(\mu-V\left\langle n_{\Lambda}^{-}\right\rangle\right)-\sum_{k=1}^{N_{\mathrm{S}^{-}}} \frac{V_{\Lambda k}^{2}}{i \omega_{n}-\epsilon_{\Lambda k}} .
$$

We use the full exact diagonalization method to calculate the impurity Green's function for this model. The number of sites $N_{S}=5$ and $N_{S}=6$ are found to be sufficient for the calculations in this paper. The free density of states is given by:

$$
D(\epsilon)=\frac{2}{\pi W^{2}} \sqrt{W^{2}-\epsilon^{2}}(|\epsilon|<W) .
$$

We set $W=1$ as the energy unit. The DMFT self-consistency equations for the Bethe lattice are the given by

$$
\mathcal{G}_{\Lambda 0}^{-1}\left(i \omega_{n}\right)_{\mathrm{dys}}=i \omega_{n}+\mu-V\left\langle n_{\Lambda}^{-}\right\rangle-\frac{W^{2}}{4} G_{\Lambda}^{-}\left(i \omega_{n}\right),
$$

where $\Lambda=A, B$. Equations (3), (4), and (6) form a set of closed self-consistent DMFT equations. After the Green's function and Weiss function are calculated, the new set of effective parameters for the impurity model is obtained via a minimization procedure: ${ }^{22}$

$$
d=\frac{1}{n_{\max }+1} \sum_{n=0, \Lambda}^{n_{\max }}\left|\mathcal{G}_{\Lambda 0}^{-1}\left(i \omega_{n}\right)_{\mathrm{map}}-\mathcal{G}_{\Lambda 0}^{-1}\left(i \omega_{n}\right)_{\mathrm{dys}}\right|^{2} .
$$

The self-consistent equations are solved iteratively. After the iteration converges, we calculate the electron densities on the two sublattices, $n_{A}$ and $n_{B}$, which in turn give the average 
electron density $n=\left(n_{A}+n_{B}\right) / 2$ and the order parameter $\mid n_{A}$ $-n_{B} \mid$. The contributions to the total energy per lattice site $E$ $=E_{T}+E_{U}+E_{V}$ from different parts of the Hamiltonian are also calculated. The kinetic energy $E_{T}$, on-site repulsion energy $E_{U}$, and the intersite repulsion energy $E_{V}$ are given by:

$$
E_{T}=\frac{1}{\beta} \sum_{n, \Lambda} \xi_{\Lambda}\left(i \omega_{n}\right) G_{\Lambda}\left(i \omega_{n}\right) e^{i \omega_{n} 0^{+}}
$$

where

$$
\begin{gathered}
\xi_{\Lambda}\left(i \omega_{n}\right)=i \omega_{n}+\mu-\mathcal{G}_{\Lambda 0}^{-1}\left(i \omega_{n}\right)(\Lambda=A, B), \\
E_{U}=\frac{1}{2 \beta} \sum_{n, \Lambda}\left[\mathcal{G}_{\Lambda 0}^{-1}\left(i \omega_{n}\right)-G_{\Lambda}^{-1}\left(i \omega_{n}\right)\right] G_{\Lambda}\left(i \omega_{n}\right) e^{i \omega_{n} 0^{+}},
\end{gathered}
$$

and

$$
E_{V}=\frac{V}{2} n_{A} n_{B}
$$

Phase separation has been studied extensively for models of strongly correlated electrons, such as the Hubbard model, ${ }^{23-25}$ the $t-J$ model, ${ }^{26,27}$ the Falicov-Kimball model, ${ }^{28}$ and the double-exchange model. ${ }^{29-31} \mathrm{~A}$ standard criterion for the PS is the discontinuous jump in the curve of electron density $n$ versus chemical potential $\mu$ (Ref. 32). However, in the DMFT study of double-exchange systems, we have observed PS also through the multiple-valued structure in a continuous $n-\mu$ curve. ${ }^{33}$ As described in Sec. III, we find in a certain temperature regime for $V=1.4$ an " $\mathrm{S}$ "- or " $\mathrm{Z}$ "-shaped multiple-valued structure in the curves of $n_{A}, n_{B}$, and $n$ versus $\mu$. Such curves contain the full information about the PS, including the metastable phase and the first-order phase transition line. To do this, we introduce ${ }^{33}$ a self-consistently determined quantity $\mu^{\prime}$ :

$$
\mu^{\prime}=\mu-\lambda\left(n_{A}-A\right),
$$

where $\lambda$ and $A$ are tunable parameters. The value of $n_{A}$ is dependent on the chemical potential $\mu$ through $n_{A}=F(\mu)$ and the functional dependence $F(\mu)$ is determined by the DMFT calculations. For a given $\mu$ and in each DMFT iteration, we first calculate $\mu^{\prime}$ and use $\mu^{\prime}$ instead of $\mu$ in the ordinary DMFT scheme to produce the local Green's function, and then extract the $\varepsilon_{\Lambda k}$ and $V_{\Lambda k}$. After that, the new value of $n_{A}$ is calculated. The iteration is carried on until convergence is reached. This is equivalent to simultaneously solve the DMFT equation and the following equation:

$$
n_{A}=F\left[\mu-\lambda\left(n_{A}-A\right)\right] .
$$

If the function $F(\mu)$ has a multiple-valued regime, by selecting appropriate parameters $\lambda$ and $A$, the $n_{A^{-}} \mu$ curve selfconsistently determined by Eq. (13) may become single valued. In this way, our calculation avoids the numerical instabilities induced by the multiple-valued structure of $n-\mu$ curve. For each $\mu$, we first solve Eq. (13) together with the DMFT equations, then calculate the thermodynamic quantities $Q$. After the data are obtained for each $\mu$, we plot the quantities $Q$ with respect to the argument $\mu^{\prime}$ to recover the physical curves $Q=F_{Q}\left(\mu^{\prime}\right)$ that correspond to the original
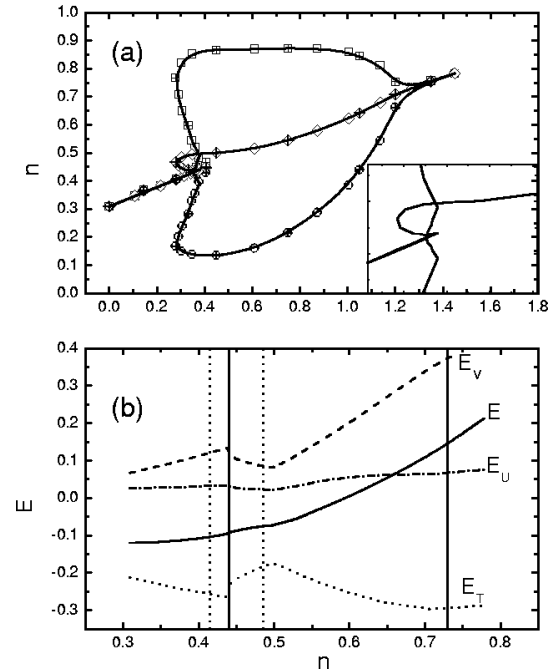

FIG. 2. Electron density $n$ vs chemical potential $\mu$ for $U=2.0$, $V=1.4$, and $T=0.05$. The squares, dots, and diamonds are for $n_{A}, n_{B}$, and $n$, respectively. Calculation results with $N_{s}=6$ (hollow symbols) and $N_{s}=5$ (cross-filled symbols) agree well. Lines are guides for the eyes. The inset is an enlarged figure for the region of the first order phase transition. (b) Contributions to the energy per lattice site as functions of $n: E_{T}, E_{V}, E_{U}$, and $E$ denote kinetic energy, nearestneighbor repulsion energy, on-site repulsion energy, and the total energy, respectively. The regime between the dotted vertical lines is unstable towards phase separation. Charge order exists between the solid vertical lines.

Hamiltonian. The final results should be independent of the parameters $\lambda$ and $A$, if only they are in an appropriate regime. In the framework of DMFT, this transformation scheme has been used in the study of PS in the double exchange model ${ }^{33}$ and of the Mott-Hubbard transition in the Hubbard model. ${ }^{34}$

\section{RESULTS AND DISCUSSION}

\section{A. Phase separation: $V=1.4>V_{c}$}

In this section, we discuss our results for $V=1.4$, which is a little larger than the zero-temperature critical value $V_{c}$ $\approx 1.32$ (see Fig. 1). For this interaction strength, as is shown in Fig. 1, the exactly quarter-filled system has a chargeordered ground state. The charge order persists up to $T$ $\approx 0.26$, and no reentrant transition was found. In order to study the system away from quarter filling, at a lower temperature $T=0.05$, we change the chemical potential and calculate charge densities. Figure 2(a) shows our result for the sublattice charge densities $n_{A}, n_{B}$, and average charge density $n$ as functions of $\mu$. As we have expected, charge order exists in some finite regime of doping around $n=1 / 2$ : $0.44<n<0.73$. This regime is not symmetric about $n=1 / 2$, since the model (1) does not have particle-hole symmetry at this point. This differs from the spinless fermion model. ${ }^{21}$ Here, the charge-ordered regime extends to larger densities, where the intersite Coulomb repulsion is more effective. However, the value of $\left|n_{A}-n_{B}\right|$ is largest at $n=1 / 2$.

One dominant feature of Fig. 2(a) is the S-shaped multiple-valued structure in the $n-\mu, n_{A^{-}} \mu$, and $n_{B^{-}} \mu$ curves. 
This is direct evidence for PS in the extended Hubbard model near quarter filling. When $n$ increases, the system first goes from a charge-disordered phase into a charge-ordered phase through a first-order transition, which occurs in the regime $n=0.4-0.5$. As $n$ increases further, the charge order disappears continuously at $n \approx 0.73$. The results obtained using $N_{s}=5$ and $N_{s}=6$ agree very well in most parts of the curve. There is only a slight deviation near the second-order transition point at $n=0.44$. This indicates that for this problem, $N_{s}=5$ is sufficient to obtain qualitatively correct conclusions. In the following, all our results are from $N_{s}=5$ calculations.

In the multiple-valued regime of the $n-\mu$ curve, the DMFT self-consistency equations have three solutions for a fixed $\mu$. We use the thermodynamical grand potential $\omega(T, \mu)$ to evaluate the relative stability of these solutions:

$$
\omega(T, \mu)=\omega\left(T, \mu_{0}\right)-\int_{\mu_{0}}^{\mu} n\left(T, \mu^{\prime}\right) d \mu^{\prime} .
$$

The actual first-order transition point $\mu_{c}$ is determined by a Maxwell construction, i.e., by solving the equation $\omega_{1}\left(T, \mu_{c}\right)=\omega_{2}\left(T, \mu_{c}\right)$. At $\mu=\mu_{c}$, two phases coexist, with their respective volumes determined by the nominal electron density of the system. At $T=0.05$, one of the coexisting phases is charge-disordered with $n_{1}=0.415$, the other one is charge-ordered with $n_{2}=0.486$. The third solution with intermediate $n$ is charge-ordered, but due to its negative compressibility and highest grand potential, it is unstable with respect to the others. For $\mu$ away from but near $\mu_{c}$, there is only one stable phase in the system, either ordered or disordered. The other two solutions have higher grand potential and are metastable. These metastable phases may be detected by hysteresis experiments.

$n=0.44$ is the lower critical density at which charge ordering occurs. At this point, the $n-\mu$ curve turns backwards sharply, and $n=-\partial \omega(T, \mu) / \partial \mu$ is continuous while $\partial n / \partial \mu$ is discontinuous. Therefore, it is identified as a second-order transition point between charge-disordered and chargeordered phases. In this respect, it is the same as the $n=0.73$ point. However, due to the higher grand potential, the second-order charge ordering transition only exists at metastable level. To understand the PS better, we show the $n$ dependence of the energy in Fig. 2(b). The on-site repulsion energy $E_{U}$ has a small contribution. It does not change much at the boundary to the charge ordering regime. This is an indication that for $U=2$, double occupancy is small in the doping range around $n \sim 0.5$, and that it plays a minor role for PS. In contrast, the kinetic energy $E_{T}$ and intersite repulsion energy $E_{V}$ are more sensitive to the charge density and long-range charge ordering. If there is no charge ordering, $E_{V}$ behaves as $E_{V} \propto n^{2}$, while $E_{T}$ recovers the behavior of the Hubbard model; this means that with increasing $n$, it decreases in the low filling area and increases near half filling.

In Fig. 2(b), it is seen that the charge order in the regime $0.44<n<0.73$ strongly reduces $E_{V}$ while it increases $E_{T}$ with respect to their value in disordered phase. The charge ordering transition at $n=0.44$ causes drastic changes in $E_{V}$ and $E_{T}$, which are then naturally related to the occurrence of

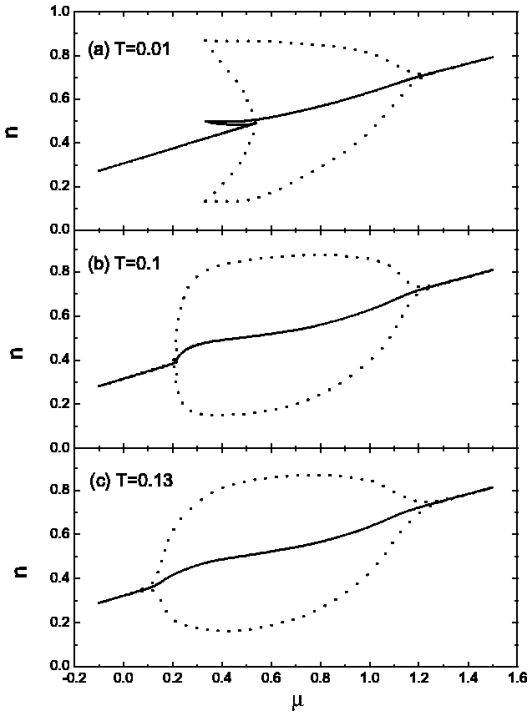

FIG. 3. Electron density $n$ vs chemical potential $\mu$ for $U=2.0$, $V=1.4$, and (a) $T=0.01$, (b) $T=0.1$, and (c) $T=0.13$. The upper and lower dotted lines and the solid line are for $n_{A}, n_{B}$, and $n$, respectively.

PS. In contrast, at the other transition point $n=0.73$, the energies change more smoothly. Because the opposite contributions from $E_{V}$ and $E_{T}$ almost cancel, the total energy $E$ increases monotonously and smoothly with increasing $n$. However, the quick change of $E_{V}$ and $E_{T}$ near $n=0.44$ and their competition leads to a small convex part in the $E$ - $n$ curve, where $\partial^{2} E / \partial n^{2}<0$. When taking into account the entropy contribution to the free energy, the convex structure will be enforced by a small amount $T S \sim 10^{-2}$. Therefore, at the temperature $T=0.05$, the $E-T$ curve already represents the behavior of free energy $F(T, n)$. The convex structure observed here is therefore consistent with PS obtained using the $n-\mu$ criterion. From Fig. 2(b), it is clear that PS is closely related to the charge ordering transition. It directly results from the charge-ordering-induced strong competition between $E_{V}$ and $E_{T}$.

In the following, we study the temperature dependence of charge order and PS. In Fig. 3, three $n-\mu$ (as well as $n_{A}-\mu$ and $n_{B^{-}} \mu$ ) isotherms are shown for $T=0.01,0.1$, and 0.13 . Up to temperature $T=0.13$, the charge order is quite robust in the intermediate density regime from $n \sim 0.3$ to $n \sim 0.7$. In contrast, PS is stable only at much lower temperatures. Compared with the $T=0.05$ curve in Fig. 2(a), the multiple-valued structure is more pronounced at $T=0.01$, while it disappears at higher temperature. As temperature decreases, the multiple-valued structure in $n$ - $\mu$ curve is compressed along the $n$ axis, but that in the $n_{A^{-}} \mu$ and $n_{B^{-}} \mu$ curves does not change much. As a result, the average electron densities of the coexisting two phases, which are determined through Maxwell construction, get closer and both move towards $n$ $=0.5$. On the other hand, as temperature increases, the multiple-valued part of the $n$ - $\mu$ curve shrinks along the $\mu$ axis until it disappears at $T \approx 0.1$ [Fig. 3(b)]. At this temperature, PS disappears and the slope of the $n-\mu$ curve diverges at the second-order charge ordering transition, leading to strong fluctuations in charge density as well as in the order 


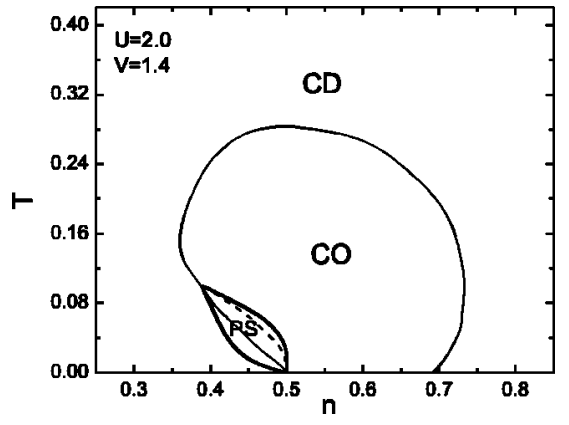

FIG. 4. Phase diagram in the $T-n$ plane for $U=2.0$ and $V=1.4$. The thin solid line is the second-order transition line. CO and CD denote charge-ordered and charge-disordered phase, respectively. PS denotes phase separation between charge-ordered and chargedisordered phases. The thick solid lines are the boundaries of the coexisting regime. In this regime, between the dashed line and the thin line lies a charge-ordered phase with negative compressibility.

parameter $\left|n_{A}-n_{B}\right|$. The critical end point of the PS is estimated to be $n \approx 0.39, T \approx 0.1$.

It is interesting to note that the low temperature behavior of PS is not unique to this model. Similar behavior has been observed in the DMFT study of first-order phase transitions in other strongly correlated electron models. The ferromagnetic-paramagnetic (FM-PM) PS in the doubleexchange model ${ }^{33}$ and the Mott-Hubbard metal-insulator transition in the half-filled Hubbard model ${ }^{34}$ are both typical first-order phase transitions. Within DMFT, they are described by continuous $\mathrm{S}$ or Z-shaped curves that are very similar with the $n-\mu$ curves presented here. As the temperature decreases, all these structures are compressed in the vertical direction. The "order parameter" of the first-order transitions $\left(\left|n_{\mathrm{FM}}-n_{\mathrm{PM}}\right|\right.$ for FM-PM PS, and double occupation difference $\left|D_{\text {met }}-D_{\text {ins }}\right|$ for the Mott-Hubbard transition) obtained from a Maxwell construction reduces to zero in the limit of $T \rightarrow 0$.

Here, though the lowest temperature that we study is $T$ $=0.01$, the main tendency is clearly that as temperature decreases, the upper two branches of the S-shaped curve [as shown in Fig. 3(a)] tend to merge. Hence, we expect that as $T \rightarrow 0$, the density difference between the two coexisting phases reduces to zero, similar with our previous findings in other systems. The two coexisting phases have the same density $n=0.5$ and the same energy. In such a scenario, the ground state of the $V=1.4$ system is singular at $n=0.5$. At this point, a charge-disordered phase and a charge-ordered phase, both with avarage density $n=0.5$, coexist in random volume proportion. An infinitesimal amount of additional holes in the system will destroy the phase-separated ground state and turn it into charge-disordered state, while electrons will turn it into charge-ordered state with $\left|n_{A}-n_{B}\right| \approx 0.7$. Again, this is different from the spinless fermion system, for which both additional holes and electrons doped into the charge-ordered ground state at $n=0.5$ cause PS. ${ }^{21}$

In Fig. 4, the $T-n$ phase diagram for $U=2.0$ and $V=1.4$ is shown. It is seen that charge order is rather robust in the filling regime around $n=0.5$. At zero temperature, the charge-ordered ground state extends from $n=0.5$ to $n$ $\approx 0.69$. This range first expands, and then shrinks as tem- perature increases, leading to a reentrant transition of charge ordering in certain doping regimes. The highest charge ordering transition temperature $T_{\mathrm{CO}, \text { max }} \approx 0.28$ is reached at $n$ $=0.5$. The PS area lies near the lower transition temperature line on the left side. When both the nominal electron filling and the temperature lie in this area, a charge-disordered phase with lower electron density will be separated from a charge-ordered phase with higher electron density. The two boundaries of this coexisting area, as shown by the solid lines in the figure, meet at two end points. The finite temperature end point is located at $\left(T_{c} \approx 0.1\right.$ and $\left.n_{c} \approx 0.39\right)$, which is easily seen for ordinary first-order phase transitions such as liquid-gas transition. The other end point is located at zero temperature ( $T=0$ and $n=0.5$ ), which is a common feature of the first-order PS described by DMFT. ${ }^{33,35}$ The second-order charge ordering transition line extends to zero temperature. Between this line (thin line in Fig. 4) and the dashed line lies an unstable area where the compressibility is negative. Metastable phases, either charge-disordered or charge ordered, exist in the two patches between the unstable area and the boundaries. They are intrinsic features of firstorder phase transitions and important for the properties of materials near PS. In doped manganites, metastable phases have been observed through the hysteresis of magnetization and resistivity with respect to temperature, ${ }^{36,37}$ as well as through the resistivity relaxation phenomenon. ${ }^{38}$ The study of metastable phases has provided valuable information about the PS in doped manganites.

Electronic PS has been studied extensively in various models such as the Hubbard model, the $t$ - $J$ model, and the double-exchange model, etc. Most of the PS scenarios discussed so far rely on the magnetic exchange mechanisms. Up to now, PS induced by pure Coulomb repulsion is observed only in the spinless fermion model. ${ }^{21}$ Our results show another example of PS caused by Coulomb repulsion only. In general, the Coulomb repulsion works against the PS, since a phase-separated state has a higher potential energy than the charge uniform state. Here we find that PS can also be driven by the charge-ordering transition which is in turn induced by pure Coulomb repulsion. When the charge orders to avoid the strong nearest-neighbor Coulomb repulsion in the high density regime, the Coulomb potential is strongly reduced which then allows for PS. It should also be noted that the PS described here exists mainly at finite temperatures, and comes from the effect of thermal fluctuations. In this respect, it is different from the ground-state PS of the spinless fermion model.

In the following we discuss the effect of long-range (beyond the nearest neighbor) Coulomb repulsion on the stability of PS between charge-disordered and charge-ordered phases. It is generally believed that long-range Coulomb repulsion will suppress a complete PS in the system, leading to an inhomogenious distribution of the electron density. Here we confine our discussion to the two-sublattice case and do not consider any incommensurate ordering. Taking into account the long-range Coulomb repulsion, the total intersite part of the Hamiltonian (including the nearest-neighbor contribution) can be formulated as 


$$
H_{\mathrm{int}}=\sum_{i, j} V_{i j} n_{i} n_{j} .
$$

In infinite dimensions, Eq. (15) reduces to its Hartree form. After performing appropriate scalings for the parameters $V_{i, j}$, the effective mean-field Hamiltonian has the form:

$$
H_{\text {int }}=\sum_{i \in A} n_{i A}\left(V_{1}\left\langle n_{B}\right\rangle+V_{2}\left\langle n_{A}\right\rangle\right)+\sum_{i \in B} n_{i B}\left(V_{1}\left\langle n_{A}\right\rangle+V_{2}\left\langle n_{B}\right\rangle\right) .
$$

The effect of long range repulsion reduces to only the nearest-neighbor type and the next-nearest-neighbor type, which are represented by $V_{1}$ and $V_{2}$, respectively. If we denote $\left\langle n_{A}\right\rangle=n+\delta$ and $\left\langle n_{B}\right\rangle=n-\delta$, then Eq. (16) further simplifies to

$$
\begin{aligned}
H_{\text {int }}= & \sum_{i}\left(V_{1}+V_{2}\right) n n_{i}+\delta\left(V_{2}-V_{1}\right) \sum_{i \in A} n_{i A} \\
& +\delta\left(V_{1}-V_{2}\right) \sum_{i \in B} n_{i B} .
\end{aligned}
$$

This means that the long-range Coulomb repulsion introduces two effects. One is the enhancement of the average repulsion as shown in the first term. The other is the frustration effect caused by the next-nearest-neighbor type repulsion. In Eq. (17) this effect is reflected by the terms proportional to $V_{2}-V_{1}$. The first term can effectively suppress the coexisting regime, but cannot destroy PS at sufficiently low temperature. ${ }^{33}$ In the low temperature limit, the density difference of two coexisting phases reduces to zero, and the average repulsion will lose its effect on the PS. In contrast, the frustration effect induced by long-range Coulomb repulsion may destroy the PS completely, since PS crucially depends on the charge-ordering transition. In particular, if the frustration is so large that the effective nearest-neighbor $V$ is less than $V_{c}$, as shown in the next section, there is no PS at all. Therefore, we conclude that the long-range Coulomb repulsion may destroy the PS through its frustration effect.

In the regime $V>V_{c}$, the charge order becomes robust with increasing $V$, as shown in Fig. 1. Therefore, we expect that as $V$ increases, the charge-ordered area in the $T-n$ phase diagram will expand in both temperature and filling regime. However, the main shape of the phase boundary will remain unchanged. In particular, due to the close relation between PS and the charge ordering transition, the PS regime may well expand while keeping its main structure.

Besides PS, we are also interested in the properties of the charge order in this regime. In Fig. 5, the order parameter $\left|n_{A}-n_{B}\right|$ is shown as a function of temperature for different fillings. Except for $n=0.5,\left|n_{A}-n_{B}\right|$ shows a nonmonotonous behavior for all the fillings we studied. It first increases and then decreases upon lowering the temperature. In the filling regime $0.5<n<0.69$ where the ground state is charge ordered, $\left|n_{A}-n_{B}\right|$ reduces to a finite value at $T=0$. For fillings outside but close to this regime, the ground state is chargedisordered and a reentrant transition occurs at finite temperature. Compared with the reentrant transition, the nonmonotonous change of the order parameter is a more general phonomenon. Experimentally, in systems with an ordered

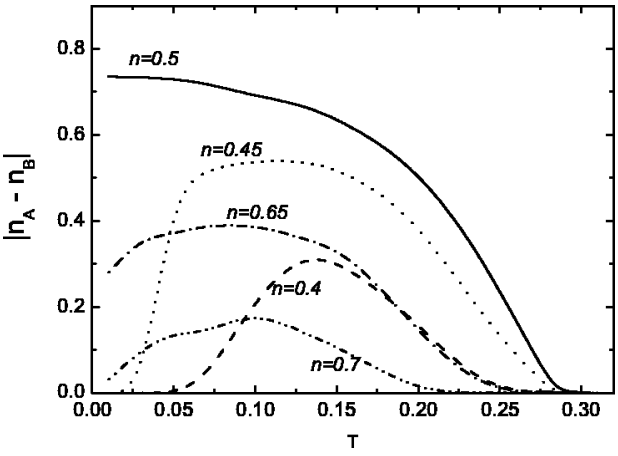

FIG. 5. The charge density difference between sublattice A and B as functions of $T$ at $U=2.0, V=1.4$ for several electron fillings: $n=0.4$ (dash), 0.45 (dot), 0.5 (solid), 0.65 (dash-dot), and 0.7 (dash-dot-dot).

ground state but near the reentrant transition, such a nonmonotonous change of the order parameter may well be observed.

\section{B. Reentrant charge ordering: $V=1.2<V_{c}$}

In this section, we discuss the case of $V=1.2$, which is smaller than $V_{c} \approx 1.32$. For this repulsion strength, the ground state of the quarter-filled system is charge-disordered. As temperature decreases, the system shows a disorderorder-disorder type reentrant transition, ${ }^{11}$ as shown in Fig. 1. When the electron filling moves away from $n=0.5$, we find that the ground state is still charge-disordered, and that a reentrant charge ordering transition exists in an extended density regime $0.42<n<0.68$. In Fig. 6, the curves for $\left|n_{A}-n_{B}\right|$ vs $T$ are shown for several fillings in this regime. It is seen that $\left|n_{A}-n_{B}\right|$ varies nonmonotonously as temperature decreases, and drops to zero at some finite temperature. Though the high temperature transition is continuous for all fillings, we find that the reentrant transition at lower temperatures has a different behavior for small and large fillings. For the filling $n=0.4$ and $n=0.45,\left|n_{A}-n_{B}\right|$ changes smoothly at the reentrant transition temperature, indicating that this transition is of second order. In contrast, in the curves for $n=0.55,0.6$, and $0.65,\left|n_{A}-n_{B}\right|$ drops abruptly near the tem-

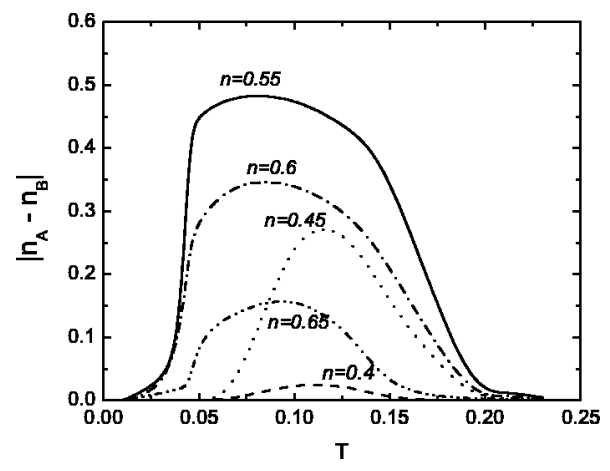

FIG. 6. The charge density difference between sublattice A and B as functions of $T$ at $U=2.0, V=1.2$ for several electron fillings: $n=0.4$ (dash), 0.45 (dot), 0.55 (solid), 0.6 (dash-dot), and 0.65 (dash-dot-dot). 


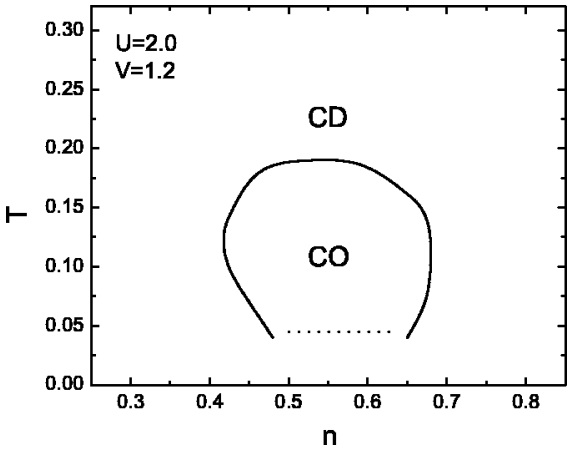

FIG. 7. Phase diagram in $T-n$ plane for $U=2.0$ and $V=1.2$. The solid line is the second-order transition line. CO and CD denote charge-ordered and charge-disordered phase, respectively. The dotted line denotes an abrupt change of the order parameter.

perature $T=0.05$. Accompanied with this rapid change of the order parameter an obvious slowing down of convergence is observed, which reduces the numerical precision significantly. A similar situation is also found for the filling $n$ $=0.5$. Since we have not found a hysteresis typical for firstorder transitions, here we would not conclude that it is a first-order phase transition. More detailed studies are needed to elucidate this issue.

For both $V=1.4$ and $V=1.2$, the nonmonotonous behavior of $\left|n_{A}-n_{B}\right|$ vs $T$ originates from the large spin entropy of the paramagnetic charge-ordered phase. For the charge-ordered phase, the twofold spin degeneracy on the occupied sites contributes a total entropy $(N / 2) \ln 2$, while for the chargedisordered state near $n=0.5$, the entropy increases from zero with increasing $T$ so at low temperature, when the chargeordered phase has a higher entropy than the disordered phase, the charge order develops as temperature increases. At higher temperature, where the entropy of the disordered phase exceeds that of the charge-ordered phase, the charge order is reduced with increasing temperature. This is the main reason that leads to the nonmonotonous behavior of the order parameter. Therefore, if the spin degeneracy of the charge-ordered phase is destroyed by the fourth-order superexchange mechanism, it is doubted whether such a reentrant behavior is still present. Hellberg et al. ${ }^{15}$ carried out a finitetemperature Lanczos study on a $4 \times 4$ lattice. They found, however, that the reentrant transition is stable when the superexchange effect is partly taken into account.

In Fig. 7, the $T-n$ phase diagram for $U=2.0$ and $V=1.2$ is shown. The reentrant charge-ordering transition exists in a regime from $n \approx 0.42$ to $n \approx 0.68$. Similar to the case of $V$ $=1.4$, the charge ordering regime is also asymmetric around $n=0.5$. Charge order appears only at finite temperatures. Here, one important difference from the $V=1.4$ diagram is that there is no PS near the reentrant transition line. For the fillings near the two vertical boundaries of the chargeordered area, the order parameter changes continuously to zero at the lower transition temperature. This continuous transition is indicated by a thick line Fig. 7. In the regime $0.5<n<0.65$, the high temperature transition is continuous, while at low temperatures, the order parameter disappears more abruptly. In the phase diagram we schematically denote such an abrupt transition by a dotted horizontal line. Due to the severe critical slowing down of convergence at this transition, it is difficult to obtain the transition temperature accurately.

Comparing Figs. 4 and 7, we see that the $T-n$ phase diagrams for $V=1.4$ and $V=1.2$ are topologically different. Near the critical $V_{c}$, the $T-n$ phase diagram, including the stability of PS and the reentrant transition, is very sensitive to $V$. When $V$ increases from $V<V_{c}$, both the $n$ and $T$ regime of the charge-ordered area expands, and the reentrant transition temperature decreases. But the ground state remains disordered, as shown in Fig. 7. At $V=V_{c}$, the ground state at $n$ $=0.5$ is expected to first turn charge-ordered. When $V$ is even larger, the filling regime of the charge-ordered ground state extends towards larger $n$, while keeping its left end-point $n$ $=0.5$ unchanged. At the same time, a PS area where chargedisordered and charge-ordered phases coexist emerges near the reentrant transition line in the $n<0.5$ regime. In this way, the phase diagram shown in Fig. 7 for small $V$ evolves into that in Fig. 4 for large $V$.

Our results are exact for the infinite dimensional extended Hubbard model on the Bethe lattice. They can also be viewed as approximate results for the models describing real materials. The effect of finite dimension and the actual lattice structure can be crudely taken into account by using the appropriate density of states and a suitable scaling of $V$ in the DMFT calculation. However, this will only lead to minor quantitative differences in the results. For a fixed lattice structure, the mean-field nature of the DMFT approach will result in transition temperatures which are somewhat overestimated as compared to the exact values. Since the temporal fluctuations in this model play an essential role, the qualitative structure of the phase diagram obtained from DMFT is expected to be valid at least for three-dimensional systems where spatial fluctuaions are weak. More precise studies of the consequences of finite dimensionality will rely on theories extending DMFT to include spatial fluctuations. ${ }^{13}$

Phenomena such as charge ordering, reentrant transition, and PS have been observed experimentally in doped manganites. The mechanisms for these phenomena are more complicated and are topics of intensive research. The interesting point here is that starting from an electronic model that only takes into account the on-site and nearest-neighbor Coulomb repulsion, we are able to obtain rich phase diagrams that include all these phenomena. Previous studies ${ }^{11}$ indicated that the occurence of a reentrant charge ordering transition does not depend on details of the localization mechanism. Here, when compared with other first-order phase transitions studied, we find that within DMFT, the structure of firstorder transitions and their temperature behavior are very similar. They are also independent of the specific mechanism. It would be interesting to do such a comparison for those real materials where different first-order phase transitions occur.

\section{SUMMARY}

In this paper, we studied the paramagnetic phase of the single band extended Hubbard model near quarter filling. In the framework of DMFT, the effective Anderson impurity 
model is solved using the exact diagonalization technique. Based on the previous $T$ - $V$ phase diagram for $n=1 / 2$ (Ref. 11 ), we concentrate on the phase diagram in $T-n$ planes for two specific value of the intersite repulsion $V: V=1.4$ and $V=1.2$, located on the two sides of the zero temperature critical value $V_{\mathrm{c}} \approx 1.32$. In both cases, charge order exists in an extended regime of filling near $n=1 / 2$. Except at some special points (such as $V=1.4$ and $n=1 / 2$ ), the order parameter of charge order $\left|n_{A}-n_{B}\right|$ changes nonmonotonously with temperature. For $V=1.4$, in the filling regime $0.39<n<0.5$ and near the reentrant transition temperature, we find PS between a charge-disordered and a charge-ordered phase. Information on this PS, including the existence of a metastable state and the first-order transition line, is described by the S-shaped structure in the continuous $n-\mu$ curve. At zero temperature, with increasing $n$, the charge-disordered ground state changes into a charge-ordered one abruptly at $n=1 / 2$. Our analysis suggests that long-range Coulomb repulsion may destroy this PS through its frustration effect. For $V=1.2$, the ground state is charge-disordered for all fillings. The reentrant charge ordering transition is observed in the regime $0.42<n<0.68$. It becomes rather abrupt in the regime $0.5<n<0.65$. Relevance of our results to the doped manganites is discussed.

\section{ACKNOWLEDGMENTS}

This work was supported by the Alexander von Humboldt Foundation (N.-H. T.), the Research Grants Council of Hong Kong (Project No. HKU7088/01P) (S.-Q. S.), and by the DFG through SFB 484 (R. B.).
${ }^{1}$ S. W. Cheong and H. Y. Hwang, in Contribution to Colossal Magneto-Resistance Oxides, Monographs in Condensed Matter Science, edited by Y. Tokura (Gordon and Breach, London, 1999).

${ }^{2}$ K. H. Kim, S. Lee, T. W. Noh, and S. W. Cheong, cond-mat/ 0203150.

${ }^{3}$ Y. Tomioka, A. Asamitsu, H. Kuwahara, and Y. Tokura, J. Phys. Soc. Jpn. 66, 302 (1997).

${ }^{4}$ T. Kimura, R. Kumai, Y. Tokura, J. Q. Li, and Y. Matsui, Phys. Rev. B 58, 11081 (1998).

${ }^{5}$ J. Q. Li, Y. Matsui, T. Kimura, and Y. Tokura, Phys. Rev. B 57, R3205 (1998).

${ }^{6}$ A. Ochiai, T. Suzuki, and T. Kasuya, J. Phys. Soc. Jpn. 59, 4129 (1990); P. Fulde, B. Schmidt, and P. Thalmeier, Europhys. Lett. 31, 323 (1995).

${ }^{7}$ M. Vojta, A. Hübsch, and R. M. Noack, Phys. Rev. B 63, 045105 (2001).

${ }^{8}$ A. Hübsch, C. Waidacher, and K. W. Becker, Phys. Rev. B 64, 241103 (2001).

${ }^{9}$ H. Mori, S. Tanaka, and T. Mori, Phys. Rev. B 57, 12023 (1998).

${ }^{10}$ J. Merino and R. H. McKenzie, Phys. Rev. Lett. 87, 237002 (2001).

${ }^{11}$ R. Pietig, R. Bulla, and S. Blawid, Phys. Rev. Lett. 82, 4046 (1999).

${ }^{12}$ M. Y. Kagan, K. I. Kugel, and K. I. Khomskii, J. Electromagn. Waves Appl. 93, 415 (2001).

${ }^{13}$ A. Georges, G. Kotliar, W. Krauth, and M. J. Rosenberg, Rev. Mod. Phys. 68, 13 (1996).

${ }^{14}$ H. A. Tuan, Mod. Phys. Lett. B 15, 1217 (2001).

${ }^{15}$ C. S. Hellberg, J. Appl. Phys. 89, 6627 (2001).

${ }^{16}$ M. Calandra, J. Merino, and R. H. McKenzie, Phys. Rev. B 66, 195102 (2002).

${ }^{17}$ A. T. Hoang and P. Thalmeier, J. Phys.: Condens. Matter 14, 6639 (2002).

${ }^{18}$ P. Sengupta, A. W. Sandvik, and D. K. Campbell, Phys. Rev. B 65, 155113 (2002); M. Tsuchiizu and A. Furusaki, Phys. Rev. Lett. 88, 056402 (2002); M. Aichhorn, H. G. Evertz, W. von der
Linden, and M. Potthoff, cond-mat/0402580.

${ }^{19}$ M. R. Ibarra, G. M. Zhao, J. M. De Teresa, B. Garcia-Landa, Z. Arnold, C. Marquina, P. A. Algarabel, H. Keller, and C. Ritter, Phys. Rev. B 57, 7446 (1998).

${ }^{20}$ H. L. Liu, S. L. Cooper, and S. W. Cheong, Phys. Rev. Lett. 81, 4684 (1998).

${ }^{21}$ G. S. Uhrig and R. Vlaming, Phys. Rev. Lett. 71, 271 (1993).

${ }^{22}$ M. Caffarel and W. Krauth, Phys. Rev. Lett. 72, 1545 (1994).

${ }^{23}$ K. Michielsen and H. De Raedt, Phys. Rev. B 59, 4565 (1999).

${ }^{24}$ P. G. J. van Dongen, Phys. Rev. Lett. 74, 182 (1995).

${ }^{25}$ R. Zitzler, Th. Pruschke, and R. Bulla, Eur. Phys. J. B 27, 473 (2002).

${ }^{26}$ V. J. Emery, S. A. Kivelson, and H. Q. Lin, Phys. Rev. Lett. 64, 475 (1990).

${ }^{27}$ S. R. White and D. J. Scalapino, Phys. Rev. B 61, 6320 (2000).

${ }^{28}$ J. K. Freericks, E. H. Lieb, and D. Ueltschi, Phys. Rev. Lett. 88, 106401 (2002); J. K. Freericks, Ch. Gruber, and N. Macris, Phys. Rev. B 60, 1617 (1999).

${ }^{29}$ S. Q. Shen and Z. D. Wang, Phys. Rev. B 58, R8877 (1998); 59, 14484 (1999); 61, 9532 (2000).

${ }^{30}$ S. Yunoki, A. Moreo, and E. Dagotto, Phys. Rev. Lett. 81, 5612 (1998).

${ }^{31}$ A. Moreo, S. Yunoki, and E. Dagotto, Science 283, 2034 (1999).

${ }^{32}$ A. Moreo, D. Scalapino, and E. Dagotto, Phys. Rev. B 43, 11442 (1991).

${ }^{33}$ N. H. Tong and F. C. Pu, Phys. Rev. B 62, 9425 (2000).

${ }^{34}$ N. H. Tong, S. Q. Shen, and F. C. Pu, Phys. Rev. B 64, 235109 (2001).

${ }^{35}$ N. H. Tong and S. Q. Shen, Mod. Phys. Lett. B 15, 1249 (2001).

${ }^{36}$ P. Schiffer, A. P. Ramirez, W. Bao, and S. W. Cheong, Phys. Rev. Lett. 75, 3336 (1995).

${ }^{37}$ N. A. Babushkina, L. M. Belova, D. I. Khomskii, K. I. Kugel, O. Yu. Gorbenko, and A. R. Kaul, Phys. Rev. B 59, 6994 (1999).

${ }^{38}$ A. Anane, J. P. Renard, L. Reversat, C. Dupas, P. Veillet, M. Viret, L. Pinsard, and A. Revcolevschi, Phys. Rev. B 59, 77 (1999). 